\title{
ON THE ORIGIN OF OSCILLATIONS IN A SOLAR DIAMETER \\ OBSERVED THROUGH THE EARTH'S ATMOSPHERE:
}

\author{
A TERRESTRIAL ATMOSPHERIC OR A SOLAR \\ PHENOMENON*
}

\author{
HENRY A. HILL, RANDALL J. BOS, and THOMAS P. CAUDELL \\ Department of Physics and Arizona Research Laboratories, University of Arizona, \\ Tucson, AZ 85721, U.S.A.
}

\begin{abstract}
Interpretations of current and past results from ground-based solar diameter measurements, as well as the planning of scientific programs for the 1980's, are strongly dependent on the perceived level of the degrading effects of the Earth's atmosphere. One of the more effective approaches has been to design the observing program and the subsequent data analysis such that the solar diameter measurements themselves could provide an evaluation of atmospheric effects. Many important results have been obtained in studies of this type and these results are collected here to help in appraising the current situation. This evidence all points in one direction: the Earth's atmosphere, while complicating the design of observational programs, is not the source of the oscillations observed in solar diameter measurements. Further, this same evidence indicates that the Earth's atmosphere will not pose any serious limitations in ground-based solar diameter studies during the 1980's.
\end{abstract}

\section{Introduction}

The signals which have been interpreted as representing solar oscillations are indeed quite small in magnitude when measured against more typical astrophysical signals. For the highest frequency resolution that has been reported to date in the observations, the whole disk velocity amplitudes are of the order of $10 \mathrm{~cm}$ per sec (Claverie et al., 1981), while the amplitudes of the diameter variations are of the order of a few milli arc sec (Bos and Hill, 1983). When the small size of these amplitudes is put into perspective, it is reasonable to expect that the presence of the Earth's atmosphere may pose problems. Various practices and techniques have been employed to reduce the effects of such problems in the measurements while numerous efforts have been undertaken to identify the residual effects in the reported results. Efforts to identify residual atmospheric effects usually may be classified as belonging to one of two classes. The first attempts to study the Earth's atmosphere through selected observations and, from this, to extrapolate to what might be expected in other observational programs. In many instances, this approach may be the only option available. However, it must be recognized that uncertainty exists in this extrapolation. Consequently, the search for these extrapolated properties in the observations may serve either to identify residual atmospheric effects in the observations, as projected, or possibly only serve to test the

\footnotetext{
* Proceedings of the 66th IAU Colloquium: Problems in Solar and Stellar Oscillations, held at the Crimean Astrophysical Observatory, U.S.S.R., 1-5 September, 1981.
}

Solar" Physics 82 (1983) 129-138. 0038-0938/83/0821-0129\$01.50.

Copyright (C) 1983 by D. Reidel Publishing Co., Dordrecht, Holland, and Boston, U.S.A. 
atmospheric model used in the extrapolation process. The actual identification of effects in the observations which match those that have been projected in the extrapolation process satisfies a necessary condition for the accuracy of the extrapolation process. A demonstration, on the other hand, that the process is correct must entail a test of the sufficient condition class and it is at this level that the following discussion of existence tests will proceed.

The second of these two classes of studies to detect residual terrestrial atmospheric effects works to identify properties present in the observations which are not consistent with those of an atmospheric source. This type of property is often difficult if not impossible to identify, but, if actually found in the observations, implies that the observed phenomenon is very probably not due to the Earth's atmosphere. Should more than one such property be identified and detected, then a considerably stronger statement can be made regarding the origin of the effect. This second class of study furnishes, to a degree, tests of a sufficient condition class. Stated more emphatically, it is sufficient to observe such a property to demonstrate that the phenomenon does not originate in the terrestrial atmosphere.

In the field of solar oscillations, observations and analyses continue to be done which fall into the necessary condition class. Since the relevance of this work depends very strongly on the degree to which results of the more powerful sufficient condition class of studies are taken into account, a summary of the currently available material in the latter category is given here. This summary, in conjunction with the various reports in the literature which primarily treat work of the necessary condition class, should provide a resource for those interpreting results, analyzing data and designing future experiments; in addition, it will serve to point out the tight constraints imposed by the results of studies that fall into the sufficient condition class.

\section{Tests of Sufficient Condition Class}

Five properties of the reported solar oscillations exist which are not consistent with the properties expected for the Earth's atmosphere and which therefore furnish the bases for a series of tests in the sufficient condition class. In each of the five cases, the placement of the origin of the oscillations in the terrestrial atmosphere would require a radical modification in our understanding of the Earth's atmosphere. These five properties of the oscillations are: a high degree of phase stability over extended periods of time; reproducibility of observed frequencies of oscillation from one data set to another; the radial dependence of the observed oscillatory signal; a direct measurement of the effect of differential refraction in the Earth's atmosphere; and spatial symmetry properties and spatial coherence of the observed oscillatory signal. These properties are also listed in Table I and have been reported in a number of papers spread over the last $\sim$ six years. These references and the salient points from these works will be brought together in the following paragraphs, culminating with the most recent results of Bos and Hill (1983). 


\section{Results Satisfying a Sufficient Condition}

Four different sets of observations made on the SCLERA $^{\dagger}$ telescope have yielded information on global oscillations of the Sun. These were obtained in 1973 (Hill and Stebbins, 1975), in 1975 (Brown et al., 1978), in 1978 (Caudell et al., 1980), and in 1979 (Bos and Hill, 1983). Each of these sets of observations of solar oscillations has, upon analysis, exhibited properties which fall into two or more of the five categories which are listed in Table I. Table II summarizes the correspondence between the original data sets and the five tests of the sufficient condition class which have been examined. Table II should also be quite useful in pointing the reader to the results of the rather extensive list of works which fall into this class.

Very important points in regard to Table II are that all work on solar diameter measurements has been included which belongs to the sufficient condition class and that tests for two or more of the five conditions have been made in each of the four sets of data. It should also be noted that a statistically significant positive result was obtained, i.e., the corresponding sufficient condition was satisfied, for all of the entries in Table II by the observations made at SCLERA.

\section{TABLE I}

List of sufficient condition tests

1. High degree of phase stability over extended periods of time.

2. Reproducibility of observed frequencies of oscillation from one data set to another.

3. The radial dependence of the observed oscillatory signal.

4. A direct measurement of the effect of differential refraction in the Earth's atmosphere.

5. Spatial symmetry properties and spatial coherence of the observed oscillatory signal.

\section{TABLE II}

Sufficient condition-type properties*

\begin{tabular}{llllll}
\hline Observations & l & 2 & 3 & 4 & 5 \\
\hline $1973(\mathrm{a})$ & $\mathrm{e}, \mathrm{f}$ & $\mathrm{e}$ & $\mathrm{e}$ & & \\
1975 (b) & $\mathrm{b}$ & & $\mathrm{b}, \mathrm{c}$ & & \\
$1978(\mathrm{c})$ & $\mathrm{c}$ & $\mathrm{g}$ & $\mathrm{c}$ & $\mathrm{g}$ & \\
$1979(\mathrm{~d})$ & $\mathrm{d}$ & $\mathrm{d}$ & $\mathrm{d}$ & & $\mathrm{d}$ \\
\hline
\end{tabular}

* The sufficient condition tests identified by the heading numbers correspond to those in Table I.
a. Hill and Stebbins (1975).
b. Brown et al. (1978).
c. Caudell et al. (1980).
d. Hill and Bos (1983).
e. Hill and Caudell (1979).
f. Caudell and Hill (1980).
g. Knapp et al. (1980).

$\uparrow$ SCLERA is an acronym for the Santa Catalina Laboratory for Experimental Relativity by Astrometry, a facility jointly operated by the University of Arizona and Wesleyan University. 


\section{A. TEMPORAL PHASE STABILITY}

Theoretical considerations indicate that low-order global oscillations of the Sun should have coherence times that are much longer than a day (Wolff, 1972). Terrestrial atmospheric waves, on the other hand, have rarely been observed to have coherence times greater than 2 or $3 \mathrm{hr}$. Exceptions to this are found in atmospheric tidal waves, which are diurnal and may be coherent over longer periods of time, and in atmospheric waves associated with storms and/or topographically special cases. The latter waves can be generated only when the atmospheric parameters are within certain very limited boundaries (Gossard and Hooke, 1975). One may argue that the SCLERA site has some sort of topography which produces coherent atmospheric waves at all times. However, it is well-documented that the weather pattern changes daily over the Santa Catalina Mountains where the SCLERA telescope is located.

There is yet another important factor. Even if a type of atmospheric wave existed which had a long coherence time, it very probably could not produce a signal in the solar diameter which also had a long coherence time. The changing observing conditions through the day are responsible for this. During an 8- or 9-hr observing day, there is a large systematic variation in the zenith angle, a change of approximately $180^{\circ}$ in the azimuth and a change of approximately $120^{\circ}$ in the paralactic angle (see Smart, 1965). A stable atmospheric wave could give rise to a temporally coherent signal in the solar diameter measurements only if each of these three angles, particularly the latter two, were constant in time, which is clearly not the case.

It should be noted that it was just this property of a long coherence time that led to the discovery of the global oscillations with periods around an hour in the 1973 observations. Hill and Stebbins (1975), in analyzing measurements of solar oblateness, averaged results from 10 days of data which covered a 17-day period. The average oblateness results were then plotted as a function of the paralactic angle in order to facilitate the identification of systematic errors introduced by the mirrors of the elevation and azimuth tracking system. The paralactic angle, $\eta$, increases monotonically during the day but the rate of increase, $\mathrm{d} \eta / \mathrm{d} t$, varies by a factor of about 2 , reaching a maximum at noon. Thus, by averaging in $\eta$ and plotting as a function of $\eta$, a procedure was performed which was equivalent to putting the data through a tuned filter whose pass band frequency scanned a range from approximately $1 / \mathrm{hr}$ to approximately $1 / 30 \mathrm{~min}$. In this way, a statistically significant oscillatory signal was detected in this frequency range which could be seen in the plot of oblateness vs $\eta$. Figure 10 in the paper by Richard (1975) shows a plot of oblateness vs $\eta$ that has not been processed to eliminate the oscillations.

It should thus come as no surprise that, when Hill and Caudell (1979) reexamined the 1973 observations, they found six oscillations that exhibited a high degree of temporal phase coherence over the 17 days; this phase coherence was found to be statistically significant (Caudell and Hill, 1980). Since the oscillations in the solar oblateness were seen in the 10 days of data analyzed by Hill and Stebbins, and since that is a number typical of the work which followed at SCLERA, the statistical 
significance of the oscillating oblateness signal vs $\eta$ should not be unlike that found for the later works.

A test for temporal phase stability was made in the same way for the 1973, 1975, and 1978 observations. For each day that observations were available, the Fourier transform was made of the data and the phase of a peak in the power density spectrum noted. These phases were then plotted as a function of time. If the coherence time of the oscillation was greater than the total length of the data set, then the plot of phases vs time would be in a straight line. In practice, the situation is not quite this simple since the actual number of oscillations between one phase determination and the next is not known. This additional complexity enters the picture in evaluating the statistical significance of the interpretation of the phase vs time data. However, this is a well defined problem and can be easily treated.

TABLE III

Temporal phase coherence*

\begin{tabular}{lclll}
\hline Observations & $\begin{array}{l}\text { Number of } \\
\text { oscillations }\end{array}$ & $\begin{array}{l}\text { Probability of } \\
\text { noncoherent } \\
\text { source** }\end{array}$ & $\begin{array}{l}\text { Probability of } \\
\text { noncoherent } \\
\text { source*** }\end{array}$ & Analysis \\
\hline 1973 (a) & 6 & 0.37 & $2.6 \times 10^{-3}$ & $\mathrm{f}$ \\
1975 (b) & 2 & 0.30 & 0.09 & $\mathrm{~b}$ \\
1978 (c) & 12 & $\begin{array}{l}0.8 \times 10^{-2} \\
\text { (average peak power extremely } \\
\text { small) }\end{array}$ & $\mathrm{c}$ \\
\hline 1979 (d) & 200 & & & $\mathrm{~d}$ \\
\hline
\end{tabular}

* The references $\mathrm{a}-\mathrm{f}$ are given in Table II.

** For individual oscillations.

*** For set of oscillations.

Table III gives average probabilities, for the first three sets of observations, that the oscillations result from noise. Since the oscillations are not coupled in any obvious way, i.e., they are not diurnal nor are they harmonics of some other frequency, they are very likely independent. The probability, then, that the complete set of oscillations for a particular data set results from noise is simply the average probability found for the individual oscillations raised to a power equal to the number of peaks. The results are given in column 4.

Sufficient additional constraints were imposed and information obtained in the 1979 observations to clearly eliminate atmospheric effects (see Bos and Hill, 1983). A phase coherence test may nevertheless be applied, and the level of noncoherent signal ascertained. The complete data set was analyzed with a frequency resolution of $1 / 41$ days or $0.28 \mu \mathrm{Hz}$, a resolution sufficient to distinguish individual eigenstates. However, in achieving this resolution, the previous tests for phase coherence are no longer viable as only one phase/oscillation is available. This problem was remedied by Bos and Hill (1983), who found that $1.1 \%$ of the power density was due to signals with coherence times $\leqslant 2$ days. 
This test shows that the coherence time for the majority of the oscillations in the 1979 observations is $\gtrsim 2$ days. By additional analysis, a coherence time $>41$ days was established by Bos and Hill (1983) for the frequency range $450-480 \mu \mathrm{Hz}$.

It would appear that temporal phase stability over periods of time numbering tens of days is a well-documented property of the oscillations in solar diameter measurements. This singular characteristic of the observations is a very important 'road sign' in the interpretation of the oscillations.

\section{B. REPRODUCIBILITY OF OBSERVED FREQUENCIES OF OSCILLATION FROM ONE DATA SET TO ANOTHER}

Global oscillations of the Sun should have well-defined frequencies since they are expected to have long coherence times (Wolff, 1972), while atmospheric phenomena are not expected to exhibit such a property. Thus, an obvious test for the origin of these oscillations is to compare one set of observations with another, examining the two spectra for peaks which are common to both. This can be done by comparing the results from one year to those of another or by breaking up one set into subsets and making a comparison. These two types of comparisons are referred to as external and internal comparisons respectively.

Both external and internal comparisons have been made involving each of the four sets of observations, the results of which are listed in Table IV. In three of the comparisons, a probability has been estimated that the number of peaks common to the

TABLE IV

Repeatability of observations*

\begin{tabular}{|c|c|c|c|c|c|c|}
\hline \multirow[t]{2}{*}{ Observations } & \multicolumn{2}{|c|}{ Internal comparison } & \multicolumn{3}{|c|}{ External comparison } & \multirow[t]{2}{*}{ Reference: } \\
\hline & $\begin{array}{l}\text { Number } \\
\text { of peaks } \\
\text { coincident }\end{array}$ & $\begin{array}{l}\text { Probability of } \\
\text { noncoherent } \\
\text { source }\end{array}$ & $\begin{array}{l}\text { Two } \\
\text { data } \\
\text { sets }\end{array}$ & $\begin{array}{l}\text { Number } \\
\text { of peaks } \\
\text { coincident }\end{array}$ & $\begin{array}{l}\text { Probability of } \\
\text { noncoherent } \\
\text { source }\end{array}$ & \\
\hline $1973(a)$ & & & 1975 & $5 / 6^{* *}$ & $\begin{array}{l}\text { no estimates } \\
\text { made }\end{array}$ & $\mathrm{e}$ \\
\hline 1975 (b) & $10 / 15$ & & & & & $\mathrm{~b}$ \\
\hline $1978(c)$ & & & 1975 & $20 /(29,36)^{* * *}$ & $9.4 \times 10^{-4}$ & $\mathrm{c}$ \\
\hline 1979 (d) & $\begin{array}{l}80 \% \text { of peaks } \\
\text { coincident } \\
\text { between } \\
400 \mu \mathrm{Hz} \\
\text { and } \\
500 \mu \mathrm{Hz}\end{array}$ & $11 \sigma$ & & & & d \\
\hline
\end{tabular}

* The references a-e are given in Table II.

** In the frequency range considered, there were six peaks in the 1973 observations and five from the 1978 observations.

*** In the frequency range considered, there were 29 peaks in the 1975 observations and 36 from the 1978 oscillations. 
spectra under consideration was the result of random noise. The new results shown in Table IV for the 1979 observations are quite important because of the statistical statements that can be derived from them, and also because, for the first time in this frequency range, probable individual eigenstates are being compared.

An interesting question, motivated by the results in Table IV, concerns why the coincidence rate is not higher than observed. In the 1973, 1975, and 1978 observations, the frequency resolution was $30 \mu \mathrm{Hz}$. It is quite apparent that this resolution is not adequate to resolve the individual eigenstates. Over time intervals of one or more years, changes in the levels of excitation of the individual eigenstates, a quite feasible occurrence, could lead to considerable changes in the structure of the power density spectra. This leads to reduced coincidence rates in external comparisons. In the case of the 1979 observations, where individual modes of oscillation are resolved, the coincidence rate is $80 \%$ for peaks in the two independent power density spectra. This is not inconsistent with what would be expected when the level of the observed noncoherent signal is taken into consideration. A similar statement applies also to the internal comparison for the 1975 observations, where approximately $67 \%$ of the peaks were found to coincide.

The results shown in Table IV do not prove that the observed oscillatory signals are global solar oscillations. However, these results are necessary if the signals are to be classified as global oscillations, and they are sufficient, to a statistically high level, to rule out random noise and, in particular, effects in the Earth's atmosphere as the origin of the signals.

\section{RADiAL DEPENDENCE OF THE OBSERVED OSCILLATORY SIGNAL}

The observed radial dependence of the oscillating component of the limb darkening function has proven to be one of the more important properties uncovered in solar diameter studies. The amplitude of the oscillatory signal, $I^{\prime}(r)$, increases rather rapidly in the last few arc sec as the limb is approached. This property, while important in discriminating between different interpretations of the observations, will also aid in improving detector design for future studies of solar oscillations and in identifying properties of the solar atmosphere not anticipated.

The manner in which the 1975 observations were performed did not permit a test for the enhancement of the oscillatory signal at the extreme limb. However, this property has been found in the 1973, 1978, and 1979 observations by Hill and Caudell (1979), Knapp et al. (1980), and Bos and Hill (1983), respectively. The existence of this property is sufficient to rule out differential refraction effects in the Earth's atmosphere.

The edge definition of the solar limb which is employed in all of these observations is extremely insensitive to seeing (Hill et al., 1975). However, the spatial properties of $I^{\prime}$ can also be used to test for this type of atmospheric effect by comparing them with those which should arise from seeing. The seeing effect is expected to produce a term that is proportional to $\mathrm{d}^{2} I / \mathrm{d} r^{2}$, where $I(r)$ is the static limb profile. The fact that the observed properties of $I^{\prime}$ are quite different from $\left(\mathrm{d}^{2} I / \mathrm{d} r^{2}\right)$ is sufficient to rule out seeing as the source of the oscillatory signals (Knapp et al., 1980). 


\section{DiRECT MEASUREMENT OF THE DIFFERENTIAL REFRACTION EFFECTS IN} SOLAR DIAMETER OBSERVATIONS

As discussed in the previous section, differential refraction, atmospheric seeing and solar oscillations produce different forms of radial dependence for the perturbations in $I(r)$. The signatures of these phenomena are sufficiently different that it is possible to measure directly the contribution of differential refraction to the solar diameter measurements.

A data analysis program was designed and implemented by Knapp et al. (1980) to identify the power density of the differential refraction effects in the solar diameter measurements. The program, based on the different radial properties described above, was applied to the 1978 observations; the power density, $P_{\text {ar }}$, in a radius measurement due to differential refraction was found to be:

$$
P_{\text {ar }}=0.25 \pm 0.09(\text { milli arc sec })^{2} / 30 \mu \mathrm{Hz}
$$

in the frequency range of $670 \mu \mathrm{Hz}$ (periods $\approx 25 \mathrm{~min}$ ). This is significantly below the power density levels found to be due to oscillations in the 1978 and the 1979 observations.

Other projections of the contribution of differential refraction to solar diameter measurements differ significantly from the results given in Equation (1). However, these projections, which fall into the class of necessary conditions, give information primarily about the extrapolation process used in making the projections. Because of this, this type of work actually falls under the study of atmospheric physics.

\section{E. SPATIAL SYMMETRY PROPERTIES AND SPATIAL COHERENCE OF OBSERVED EIGENFUNCTIONS}

The observations obtained by Bos and Hill (1983) were made in such a way as to yield information on changes in the limb darkening function at six different locations on the perimeter of the solar disk. These six independent signals have been analyzed for certain spatial properties of the oscillatory signals. It was observed that the eigenfunctions are spatially global and, to an accuracy of better than $20 \%$, either symmetric or antisymmetric for reflection on the solar disk through the Sun's center and for reflection about the solar equator.

So, for the majority of the oscillations observed in 1979 , a distinct set of symmetry properties may now be defined. With respect to identifying the origin of the oscillations, an equally important result is that their symmetry axes coincide, within the observational error, with the rotational axis of the Sun.

The observed spatial properties place very strong constraints on the types of random atmospheric effects which may contribute to the observations. It would be quite difficult for atmospheric seeing, with a typical aplanatic patch size of $\approx 10 \mathrm{arcsec}$, to produce oscillatory signals at diametrically opposite edges of the Sun that are highly correlated. Even more discriminating are the observed spatial symmetries, which are either symmetric or antisymmetric for reflections through the center of the solar disk and reflections about the solar equator. Only an extremely complicated epicycle model of the Earth's atmosphere could give rise to such properties. 
It should also be noted that if the atmosphere were the origin of the oscillations, then any observatory near to SCLERA must observe a different set of spatial properties and, in particular, not the symmetric and antisymmetric ones observed at SCLERA. In other words, to insist that the atmosphere is the source requires that the SCLERA telescope be at a unique spot on the Earth.

\section{Summary}

Investigations framed as sufficient tests for atmospheric effects in solar diameter measurements have been reviewed in this paper. It is important to note that no filtering has been made in gathering this material. These investigations were designed such that the demonstrated presence of a specific property or combination of properties in the observations would be sufficient to rule out terrestrial atmospheric effects as a source of the oscillatory signals in the solar diameter measurements. In each instance where a test of this nature was performed, a statistically significant result was obtained which does not support the interpretation of the observed oscillatory signals as arising from atmospheric effects and/or random noise. These results furnish very important clues as to the nature of the oscillations. Furthermore, any proposal put forward for the interpretation of the oscillations in solar diameter measurements should be subjected to nothing less than the tests made possible by these research results.

\section{Acknowledgements}

We wish to acknowledge valuable discussions with Jerry Logan and to thank Philip Goode for a critical reading of the manuscript. This work was supported in part by the Astronomy Division of the National Science Foundation and by the Air Force Office of Scientific Research.

\section{References}

Bos, R. J. and Hill, H. A.: 1983, Solar Phys. 82, 89 (this volume).

Bown, T. M., Stebbins, R. T., and Hill, H. A.: 1978, Astrophys. J. 223, 324.

Caudell, T. P. and Hill, H. A.: 1980, Monthly Notices Roy. Astron. Soc. 193, 381.

Caudell, T. P., Knapp, J., Hill, H. A., and Logan, J. D.: 1980, in H. A. Hill and W. A. Dziembowski (eds.), Nonradial and Nonlinear Stellar Pulsation, Lecture Notes in Physics, No. 125, Springer-Verlag, Berlin, p. 206.

Claverie, A., Isaak, G. R., McLeod, C. P., van der Raay, H. B., and Roca Cortes, T.: 1981, Nature $293,443$. Gossard, E. E. and Hooke, W. H.: 1975, Waves in the Atmosphere, Developments in Atmospheric Sciences, No. 2, Elsevier Scientific Publishing Co., Amsterdam, Oxford, New York.

Groth, E. J.: 1975, Astrophys. J. Suppl. 29, 286.

Hill, H. A. and Caudell, T. P.: 1979, Monthly Notices Roy. Astron. Soc. 186, 327.

Hill, H. A. and Stebbins, R. T.: 1975, Astrophys. J. 200, 471

Hill, H. A., Stebbins, R. T., and Oleson, J. R.: 1975, Astrophys. J. 200, 484.

Knapp, J., Hill, H. A., and Caudell, T. P.: 1980, in H. A. Hill and W. A. Dziembowski (eds.), Nonradial and Nonlinear Stellar Pulsation, Lecture Notes in Physics, No. 125, Springer-Verlag, Berlin, p. 394. 
Richard, J.-P.: 1975, in G. Shaviv and J. Rosen (eds.), General Relativity and Gravitation, John Wiley and Sons, New York, Toronto, and Israel Universities Press, Jerusalem, p. 169.

Smart, W. M.: 1965, Textbook on Spherical Astronomy, 5th edition, Cambridge University Press, Cambridge, p. 49.

Wolff, C. L.: 1972, Astrophys. J. 176, 833. 